\title{
Meteorological Conditions Related to the Onset of Spontaneous Pneumothorax
}

\author{
Berkant ÖZzPolat, ${ }^{1}$ Alper GözübüyüK, ${ }^{2}$ Bülent KoÇER, ${ }^{3}$ RaSiH Yazkan, ${ }^{4}$ \\ Koray DURAL ${ }^{3}$ and ONur Genç ${ }^{2}$ \\ ${ }^{1}$ Department of Thoracic Surgery, Kırıkkale University, School of Medicine, Kırıkkale, Turkey \\ ${ }^{2}$ Department of Thoracic Surgery, Gülhane Military Medical Academy, Ankara, Turkey \\ ${ }^{3}$ Department of Thoracic Surgery, Numune Training and Research Hospital, Ankara, Turkey \\ ${ }^{4}$ Department of Emergency, Ankara Yıldırım Beyazıt Training and Research Hospital, Ankara, Turkey
}

Spontaneous pneumothorax is defined as the rupture of bleb or emphysematous bullae that develop just beneath the pulmonary pleura. Weather changes may influence the incidence of spontaneous pneumothorax. The aim of this study was to examine the influence of rainfall, temperature and atmospheric pressure changes on the onset of spontaneous pneumothorax. The study involved 669 spontaneous pneumothorax admissions to three reference hospitals in Ankara, Turkey between 1996 and 2006 (612 males and 57 females with the mean age of $34.0 \pm 15.5$ years). The meteorological data were obtained from Turkish State Meteorological Services for temperature, atmospheric pressure, and rainfall. The correlation between these values and spontaneous pneumothorax clusters, which was defined as the admission of at least two patients with pneumothorax within three days of each other, was evaluated. Among 669 episodes of spontaneous pneumothorax, 472 (70.5\%) occurred in 188 clusters. When compared to days without spontaneous pneumothorax, the amount of average rainfall on the day of admission with spontaneous pneumothorax, one day before and two days before the admission was significantly high. Similarly, the atmospheric pressure on one day and two days before the admission of spontaneous pneumothorax patients was significantly low. In addition, maximum temperature level was significantly lower on admission day of spontaneous pneumothorax patients compared to those on the days without spontaneous pneumothorax. This largest series of the literature shows that spontaneous pneumothorax occurs in clusters and suggests that rainfall, temperature and falls in atmospheric pressure might play a role in the pathophysiology of spontaneous pneumothorax. — Spontaneous pneumothorax; atmospheric pressure; temperature; clustering; rainfall.

Tohoku J. Exp. Med., 2009, 217 (4), 329-334. (C) 2009 Tohoku University Medical Press

Spontaneous pneumothorax (SP) is defined as the rupture of bleb or emphysematous bullae that develop just beneath the pulmonary pleura (Suarez-Varel et al. 2000). Primary pneumothorax occurs without an underlying lung disease and the incidence is shown to be higher in young, tall, and weak men (i.e. low body mass index), smokers. Secondary pneumothorax occurs over the background of pre-existing disease (Smit et al. 1999; Huang et al. 2007). The cause of the rupture of blebs or bullae remains unclear, but it is suggested that rupture occurs when a substantial transpulmonary pressure gradient is present (Smit et al. 1999). Trapped air in the alveoli will expand when the out of body pressure falls, which accounts for pneumothorax that occurs in flying personnel or scuba divers (Dermksian and Lamb 1959; Raymond 1995; Smit et al. 1999).

Association of SP and changes in meteorological con- ditions has been investigated. Atmospheric pressure (AP), temperature changes, or correlation with specific weather phases was suggested to be precipitating factors in the development of SP (Bense 1984; García et al. 1985; Smit et al. 1999; Bulajich et al. 2005; Alifano et al. 2007). Association between SP and storm was also found (Smit et al. 1999; Alifano et al. 2007).

This study aimed to examine whether variations in meteorological conditions could be a factor in the development of SP. A discussion comparing the results of other studies based on the same hypothesis was presented.

\section{Patients and Methods}

This retrospective study consisted of $669 \mathrm{SP}$ admissions to three reference hospitals between 1996 and 2006 in Ankara, Turkey. Patients with primary SP and SP associated with chronic obstructive

Received February 13, 2009; revision accepted for publication March 12, 2009.

Correspondence: Berkant Özpolat, M.D., Department of Thoracic Surgery, School of Medicine, Kırıkkale University, Süleyman Demirel

Training and Research Hospital, Sağlık Sk., Fabrikalar Mah., Kırıkkale, Turkey, 71100.

e-mail: berkantozpolat@yahoo.com 
pulmonary disease (COPD) were included to the study. All cases were diagnosed and managed in the emergency services of the hospitals. The exclusion criteria were as follows: (a) presence of iatrogenic, traumatic pneumothorax or secondary SP other than COPD; (b) referrals from others hospitals located outside the area of the study. There were 669 episodes of SP: 582 cases of primary SP and 87 cases of SP associated with COPD. The patients were 612 males and 57 females, and the mean age was $34.0 \pm 15.5$ years, with extremes of 14 and 79 years.

Pneumothorax was diagnosed based on the chest radiography findings. In the study of Scott et al. (1989) instead of time of admission the onset of symptoms was considered as the index day, however like in our study, Bense (1984) considered the time of admission for the index day. Because of the retrospective character of the patient history study, the day that SP developed was not considered to be reliable enough to define. Therefore we decided to compare the measured meteorological values of the two consecutive days prior to admission as well as the actual day of admission. As previously reported, a cluster of pneumothorax cases was defined as the admis- sion of at least two patients with pneumothorax within 3 days of each other (Smit et al. 1997; Boulay et al. 1998; Alifano et al. 2007). The study was approved by the institutional committee of Ankara Yıldırım Beyazıt Training and Research Hospital.

\section{Meteorological Data Collection}

The meteorological data were obtained from Turkish State Meteorological Services. The data obtained from the local meteorological institute is located at the same area as the participating hospitals, within a 48-km-wide area and all patients lived within $48 \mathrm{~km}$ of the city. Analysis of the topography showed within this area the ground elevations above see level did not vary. The AP (mbar) was thus taken to be the same for all patients in the study. Climatic variable measurements of years 1996 to 2006 were provided. The mean, maximum and minimum temperature $\left({ }^{\circ} \mathrm{C}\right)$, mean, maximum and minimum AP (mbar), and rainfall ( $\mathrm{mm}$ ) were included for each study day. Calculations were made to assess differences in the values of rainfall, $\mathrm{AP}$, and temperature between each study day (D) and the day before (D-1) and two days before (D-2). Wider AP and temperature varia-

A

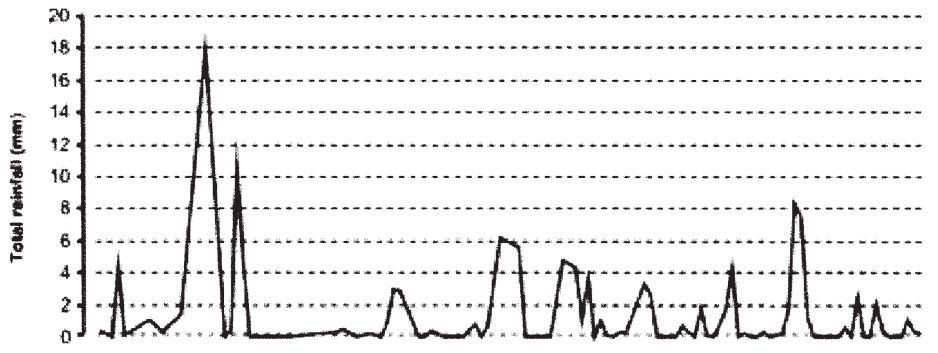

B
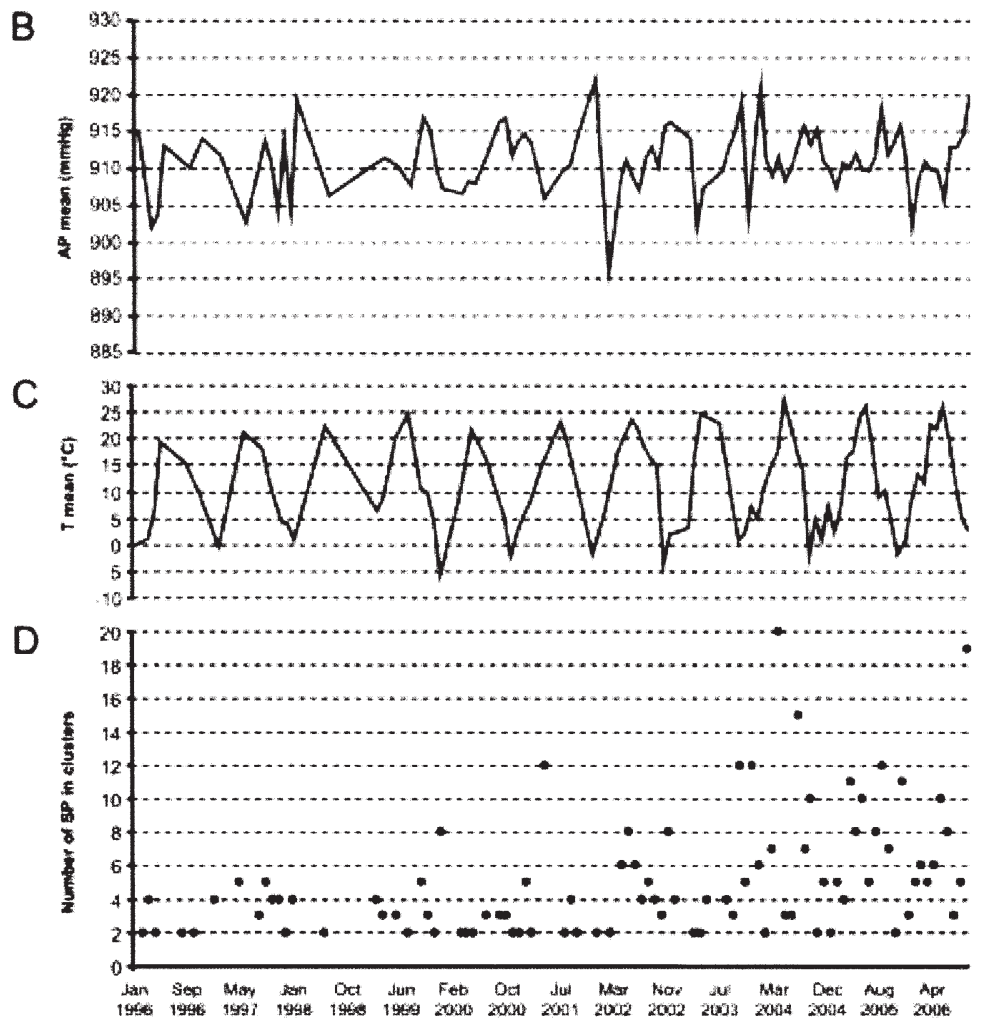

Fig. 1. Effects of meteorological conditions on the onset of of spontaneous pneumothorax.

A: Total rainfall during the study. B: APmean during the study. C: Tmean during the study. D: Occurence of clusters (Dots indicate the number of pneumothorax during the cluster). 
tions as; maximum values of the considered day minus minimum values of the D-1 and D-2 and vice versa were also calculated.

\section{Statistical analysis}

Statistical analysis was performed using Statistical Package for Social Sciences (SPSS) 11.5 software (SPSS Inc., Chicago, IL, United States). Whether the continuous data were normally distributed or not was determined by using Shapiro Wilk test. Continuous variables were expressed as mean \pm standard deviation. The differences between days with pneumothorax and days without pneumothorax for the values of rainfall, AP, and temperature were evaluated by Mann Whitney U test. A p value less than 0.05 was considered statistically significant.

\section{Results}

\section{Occurrence of SP in Clusters}

Among 669 episodes of SP, $472(70.5 \%, 95 \% \mathrm{CI}$ : 67.1-74.0) occurred in 188 clusters (Fig. 1). The mean number of pneumothorax episodes per cluster was $2.5 \pm 0.8$ (min: 2, max: 7).

TABLE 1. Rainfall on days without SP and days with SP.

\begin{tabular}{lccc}
\hline $\begin{array}{c}\text { Total rainfall } \\
(\mathrm{mm})\end{array}$ & Days without SP & Days with SP & $P$ \\
\hline D & $0.4 \pm 1.50$ & $1.5 \pm 5.56$ & $<0.001$ \\
D-1 & $0.5 \pm 1.92$ & $1.3 \pm 3.48$ & $<0.001$ \\
D-2 & $0.4 \pm 1.06$ & $1.6 \pm 5.87$ & $<0.001$ \\
D - (D-1) & $-0.1 \pm 2.36$ & $0.2 \pm 6.30$ & 0.973 \\
D - (D-2) & $0.03 \pm 1.61$ & $-0.1 \pm 7.91$ & 0.966 \\
\hline
\end{tabular}
before

\section{Rainfall}

The total rainfall values on D, D-1 and D-2 for pneumothorax days were significantly higher than the total rainfall values on the non-pneumothorax days ( $p<0.001, p<$ 0.001 , and $p<0.001$, respectively) (Table1).

\section{Atmospheric Pressure}

The AP values on D-1 and D-2 for pneumothorax days were significantly lower than the AP values on the nonpneumothorax days $(p=0.002$ and $p<0.001)$. The maximum AP values on D-1 and D-2 for the pneumothorax days were significantly lower than the AP values on the nonpneumothorax days $(p=0.002$ and $p<0.001)$. Similarly, the minimum AP values on D-1 and D-2 for the pneumothorax days were significantly lower than the AP values on the non-pneumothorax days $(p=0.006$ and $p<0.001)$.

The rates of change in the minimum AP on D-1 and D-2 and in the maximum AP on D for the pneumothorax days were significantly higher than those for the non-pneumothorax days $(p=0.004$ and $p=0.009)$. The rates of change in the maximum AP on D-1 and D-2 and in the minimum AP on D for the pneumothorax days were significantly lower than those for the non-pneumothorax days ( $p=$ 0.006 and $p=0.012$ ) (Table 2).

\section{Temperature}

The maximum temperature on $\mathrm{D}$ for the days without pneumothorax episodes was higher than that on the pneumothorax days $(p=0.039)$. The difference between the maximum and minimum temperatures on $\mathrm{D}$ for the nonpneumothorax days was significantly higher than that for the pneumothorax days $(p<0.001)$. The difference between the temperature on D-2 and the temperature on D

TABLE 2. AP on days without SP and days with SP.

\begin{tabular}{lrrr}
\hline Atmospheric Pressure (mb) & Days without SP & Days with SP & \multicolumn{1}{c}{$p$} \\
\hline APmean D & $913.9 \pm 4.85$ & $913.4 \pm 5.23$ & 0.343 \\
APmean (D-1) & $914.4 \pm 4.47$ & $913.1 \pm 4.95$ & 0.002 \\
APmean (D-2) & $914.3 \pm 4.28$ & $912.9 \pm 5.05$ & $<0.001$ \\
APmax D & $915.2 \pm 4.64$ & $914.6 \pm 5.05$ & 0.475 \\
APmax (D-1) & $915.7 \pm 4.41$ & $914.4 \pm 4.89$ & 0.002 \\
APmax (D-2) & $915.6 \pm 4.21$ & $914.3 \pm 4.98$ & $<0.001$ \\
APmin D & $912.9 \pm 5.08$ & $912.3 \pm 5.62$ & 0.506 \\
APmin (D-1) & $913.3 \pm 4.64$ & $912.1 \pm 5.17$ & 0.006 \\
APmin (D-2) & $913.3 \pm 4.48$ & $911.9 \pm 5.25$ & $<0.001$ \\
APmax D-APmin D & $2.5 \pm 1.51$ & $2.9 \pm 1.55$ & 0.480 \\
APmean D - APmean (D-1) & $-0.4 \pm 2.72$ & $0.2 \pm 3.22$ & 0.003 \\
APmean D - APmean (D-2) & $-0.3 \pm 4.66$ & $0.3 \pm 5.02$ & 0.022 \\
APmax D - APmin (D-1) & $1.9 \pm 2.80$ & $2.5 \pm 3.12$ & 0.004 \\
APmax D - APmin (D-2) & $1.9 \pm 4.67$ & $2.7 \pm 4.97$ & 0.009 \\
APmax (D-1) - APmin D & $-0.3 \pm 3.12$ & $-0.2 \pm 3.64$ & 0.006 \\
APmax (D-2) - APmin D & $2.7 \pm 4.90$ & $1.9 \pm 5.29$ & 0.012 \\
\hline
\end{tabular}

AP, Atmospheric pressure; D, Admission day; D-1, One day before; D-2, Two days before 
TABLE 3. Temperature on days without SP and days with SP.

\begin{tabular}{lcrr}
\hline \multicolumn{1}{c}{ Temperature $\left({ }^{\circ} \mathrm{C}\right)$} & Days without SP & Days with SP & \multicolumn{1}{c}{$p$} \\
\hline Tmean D & $13.4 \pm 8.97$ & $12.0 \pm 8.64$ & 0.082 \\
Tmean (D-1) & $13.2 \pm 9.15$ & $12.2 \pm 8.55$ & 0.150 \\
Tmean (D-2) & $13.0 \pm 9.38$ & $12.3 \pm 8.46$ & 0.309 \\
Tmax D & $19.1 \pm 9.86$ & $17.4 \pm 9.59$ & 0.039 \\
Tmax (D-1) & $18.9 \pm 10.10$ & $17.6 \pm 9.62$ & 0.125 \\
Tmax (D-2) & $18.6 \pm 10.33$ & $17.8 \pm 9.69$ & 0.266 \\
Tmin D & $7.6 \pm 7.91$ & $6.9 \pm 7.60$ & 0.282 \\
Tmin (D-1) & $7.6 \pm 8.10$ & $7.0 \pm 7.36$ & 0.300 \\
Tmin (D-2) & $7.0 \pm 8.20$ & $7.0 \pm 7.29$ & 0.810 \\
Tmax D - Tmin D & $11.4 \pm 3.90$ & $10.5 \pm 3.64$ & $<0.001$ \\
Tmean D - Tmean (D-1) & $0.2 \pm 2.26$ & $-0.2 \pm 2.30$ & 0.122 \\
Tmean D - Tmean (D-2) & $0.4 \pm 3.05$ & $-0.3 \pm 3.37$ & 0.014 \\
Tmax D - Tmin (D-1) & $11.5 \pm 4.46$ & $10.4 \pm 4.57$ & 0.005 \\
Tmax D - Tmin (D-2) & $10.9 \pm 3.86$ & $10.9 \pm 4.23$ & 0.940 \\
Tmax (D-1) - Tmin D & $11.2 \pm 3.62$ & $10.7 \pm 3.48$ & 0.040 \\
\hline
\end{tabular}

T, Temperature; D, Index day; D-1, One day before; D-2, Two days before

for the non-pneumothorax days was significantly higher than that for the pneumothorax days. The rates of change in the minimum temperature on D-1 and in the maximum temperature on $\mathrm{D}$ for the non-pneumothorax days were significantly higher than those for the pneumothorax days ( $p=$ 0.005 ). The rates of change in the maximum temperatures on D-1 and D-2 and in the minimum temperature on D for the pneumothorax days were significantly lower than those for the non-pneumothorax days $(p=0.040$ and $p<0.001)$ (Table 3).

Fig. 1 also illustrates trends in the mean AP, mean temperature, and total rainfall throughout the study.

\section{Discussion}

In the fifth century B.C., Hippocrates suggested that weather changes might play a role in the deterioration of physical health (Danielides et al. 2002). Climatic characteristics, such as rain, AP, thunderstorms, temperature, weather phases, and wind speed have been repeatedly implicated in the pathogenesis of many diseases. Such associations have been suggested for acute gouty arthritis, rheumatoid arthritis, SLE, Behçet's disease, Bell's palsy, sudden hearing loss, myocardial infarction and coronary deaths, abdominal aortic aneurysm ruptures, asthma, some of which showed a significant relationship (Celenza et al. 1966; Danet et al. 1999; Danielides et al. 2002; Kurtoglu et al. 2004). Similarly, this relationship has been investigated for SP, and different theories have evolved to explain the pathogenesis of this disorder.

This study estimated the impact of meteorological events on occurrence of SP in Ankara. When compared to most previous studies, it consists of the largest number of cases for the longest period. The study included a variety of meteorological parameters similar to those in some previous reports.

Our study shows that SP occurs in clusters, confirming other series (Smit et al. 1997; Boulay et al. 1998; Smit et al. 1999; Bulajich et al. 2005; Alifano et al. 2007). Alifano et al. (2007) reported that $84 \%$ of SP was admitted in clusters, and this value was $73 \%$ in other report (Smit et al. 1999). We used the same definition for an appropriate comparison and found that $70.5 \%$ of the patients applied in clusters. In each cluster, the mean number of pneumothorax episodes was $2.5 \pm 0.8$ (min: 2-max: 7). Boulay et al. (1998) and Alifano et al. (2007) reported these values as 2.7 and 3.2 respectively, which are similar to those found in our study.

The incidence of SP had no seasonal or monthly correlation in our study. Similarly no seasonal or monthly predominance of clusters could be identified in some studies (Smit et al. 1999; Bulajich et al. 2005; Alifano et al. 2007). However, one study has demonstrated slight spring preponderance and a significant increase in the rate of SP in May, and another one in summer and in July (Suarez-Varel et al. 2000; Ayed et al. 2006). Ozenne et al. (1984) demonstrated two peaks of frequency, one in summer and the other one in winter, and a significant correlation with low humidity. Thus, the authors suggested that bronchoconstriction induced by moistening of air in the airways might play a role in the pathophysiology of pneumothorax. Besides other meteorological conditions, the consequences of AP on respiratory disease have been studied frequently besides other meteorological conditions (Schmerber and Manderlier 1979; Bense 1984; Ozenne et al. 1984; Celenza et al. 1996; Smit et al. 1999; Suarez-Varel et al. 2000; Bulajich et al. 2005; Alifano et al. 2007). Alifano et al. (2007) found no correlations between pneumothorax and variations in AP fall SP. However, there was significant correlation with wider differences in AP mean between the index day and 
the previous day, and between the AP minimum and the AP maximum on the previous day. Occurrence of clusters during or immediately following drops in AP specifically occurred (Alifano et al. 2007). Bense (1984) found that a fall in AP of at least 10 millibars within 24 hours was followed by a significant increase in the number of hospital admissions 48 hours later. In contrast, some authors found no correlations between AP variations and occurrence of SP (Schmerber and Manderlier 1979; Smit et al. 1999; SuarezVarel et al. 2000; Bulajich et al. 2005). A comparison between these studies is difficult because of different study designs, study populations, different climatic conditions of countries, and small number of patients. In our experience, clusters of SP episodes were associated with falls in AP mean, AP maximum, and AP minimum in one and two days before the index day. In addition, there is a significant association between wider differences in AP minimum of the one and two days before the index day and AP maximum of the index day. Similarly, there was a significant association between AP maximum of the one and two days before the index day and AP minimum of the index day. The exact mechanism by which a fall in AP may cause SP remains unknown. A suggested mechanism states that when the air inside the blebs or bullae is trapped due to bronchospasm, a rapid equilibration of the pressure gradient with the surrounding atmosphere could not be achieved (Smit et al, 1999; Alifano et al. 2007). Another suggested mechanism was like the mechanism in asthma, where there may be a relation between inflammation and weather conditions; thus, a check valve mechanism may result in SP (Smit et al. 1997). Similarly our study suggests that transpulmonary pressure gradient due to falls in AP may be enough to cause pneumothorax in some patients. In the literature, the changes in pressure have been shown to cause pneumothorax in divers and flying personnel (Dermksian and Lamb 1959; Raymond 1995). Besides these important changes in pressure, small variations, such as loud music is also reported to cause SP (Noppen et al. 2004).

In our study, we found a significant correlation between days with SP and rain. Total rainfall was significantly high on the index day and one and two days before the index day. Alifano et al. (2007) found a significant association between pneumothorax and days on which storms occurred. They defined the storm as a heavy rainfall along with thunder and lightening. Smit et al. (1999) found similar results and speculated that various other factors such as changes in humidity, pollen concentration, air pollution, electrostatic power, as well as physical stress, were associated with storms. Our meteorological data did not include such parameters thus; their associations with SP occurrence could not be studied. With regard to asthma, recent studies suggest that mist and fog (i.e. air borne water droplets) may be a stimulus for bronchoconstriction, which has been also suggested to play a role in the occurrence of SP (Ozenne et al. 1984; Bulajich et al. 2005).

Most studies analyzing the AP changes also considered temperature changes. Some authors found no relation between SP and variations in atmospheric temperature (Ozenne et al. 1984; Bulajich et al. 2005; Alifano et al. 2007). However Smit et al. (1999) found a small but a significant temperature increase on the day before the onset of pneumothorax, whereas a small drop in temperature was found on non-pneumothorax days. In our study, similarly, a significant association could be identified between temperature and occurrence of SP, but contrary to the findings by Smit et al. (1999), a small drop in maximum temperature was determined on the index day. The discrepancies between the two studies may be due to the difference in the number of cases. We believe that this temperature fall on the index day is a cofactor for SP occurrence and should be studied in the future.

The main limitation of this study was the lack of detail on the clinical histories (smoking history) and conditions of the patients. This prevented us from confirming the diagnosis. Therefore we decided to compare the measured meteorological values of the two consecutive days prior to admission as well as the actual day of admission. Some previous studies included more consecutive days prior to admission but investigated relatively a very few number of patients comparing to our study. Although we examined 2 consecutive days, this study contained the largest study population in the literature. Furthermore, several meteorological parameters were assessed (variations of mean and maximal values of rainfall, temperature, and AP between the index day and the previous 3 days).

As a conclusion this study containing the largest series of the literature shows that SP occurs in clusters and demonstrates a significant relationship between rainfall, temperature, and fall in AP. We suggest that these changes might play a role in the pathophysiology of SP. Other studies in different countries based on similar designs should be developed to further confirm and detail the influences of climatic changes on SP occurrence.

\section{Acknowledgments}

We would like to thank the Turkish State Meteorological Services for providing the weather data and Serap Akgündüz, İlkay Kocaman, Zerrin Demirörs for the preparation of the data, and Salih Ergöçen for his valuable contributions to statistical analysis.

\section{References}

Alifano, M., Forti Parri, S.N., Bonfanti, B., Arab, W.A., Passini, A., Boaron, M. \& Roche, N. (2007) Atmospheric pressure influences the risk of pneumothorax: beware of the storm! Chest, 131, 1877-1882.

Ayed, A.K., Bazerbashi, S., Ben-Nakhi, M., Chandrasekran, C., Sukumar, M., Al-Rowayeh, A. \& Al-Othman, M. (2006) Risk factors of spontaneous pneumothorax in Kuwait. Med. Princ. Pract., 15, 338-342.

Bense L. (1984) Spontaneous pneumothorax related to falls in atmospheric pressure. Eur. J. Respir. Dis., 65, 544-546.

Boulay, F., Sisteron, O., Chevallier, T. \& Blaive, B. (1998) Predictable mini-epidemics of spontaneous pneumothorax: haemop- 
tysis too? Lancet, 351, 522.

Bulajich, B., Subotich, D., Mandarich, D., Kljajich, R.V. \& Gajich, M. (2005) Influence of atmospheric pressure, outdoor temperature, and weather phases on the onset of spontaneous pneumothorax. Ann. Epidemio., 15, 185-190.

Celenza, A., Fothergill, J., Kupek, E. \& Shaw, R.J. (1996) Thunderstorm associated asthma: a detailed analysis of environmental factors. BMJ., 312, 604-607.

Danet, S., Richard, F., Montaye, M., Beauchant, S., Lemaire, B., Graux, C., Cottel, D., Marecaux, N. \& Amouyel, P. (1999) Unhealthy effects of atmospheric temperature and pressure on the occurrence of myocardial infarction and coronary deaths. A 10-year survey: the Lille-World Health Organization MONICA project (Monitoring trends and determinants in cardiovascular disease). Circulation, 100, E1-7.

Danielides, V., Nousia, C.S., Bartzokas, A., Lolis, C.J., Kateri, M. \& Skevas A. (2002) Weather conditions and sudden sensorineural hearing loss. BMC Ear Nose Throat Disord., $2,2$.

Dermksian, G. \& Lamb, L.E. (1959) Spontaneous pneumothorax in apparently healthy flying personnel. Ann. Intern. Med., 51, 39-51.

García, J.A., Hernández, M.A., Rego, G. \& Bustillo, E. (1985) Association between falls in atmospheric pressure and spontaneous pneumothorax. Eur. J. Respir. Dis., 66, 230.

Huang, T.W., Cheng, Y.L., Tzao, C., Hung, C., Hsu, H.H., Chen, J.C. \& Lee, S.C. (2007) Factors related to primary bilateral spontaneous pneumothorax. Thorac. Cardiovasc. Surg., 55, 310-312.

Kurtoglu, M., Yanar, H., Aksoy, M., Ertekin, C., Tunca, F.,
Güloğlu, R. \& Tinay, I. (2004) Seasonality in the incidence of abdominal aortic aneurysm ruptures: a review of eight years. Ulus. Travma Acil Cerrahi Derg., 10, 39-41.

Noppen, M., Verbanck, S., Harvey, J., Van Herreweghe, R., Meysman, M., Vincken, W. \& Paiva, M. (2004) Music, a new cause of primary spontaneous pneumothorax. Thorax, 59, 722-724.

Ozenne, G., Poignie, P., Lemercier, J.P., Nouvet, G. \& Grancher, G. (1984) Meteorological conditions and spontaneous pneumothorax. Retrospective study of 165 cases in the Rouen area. Rev. Pneumol. Clin., 40, 27-33.

Raymond LW. (1995) Pulmonary barotraumas and related events in divers. Chest, 107, 1648-1652.

Schmerber, J.M. \& Manderlier, T.G. (1979) Relationship between atmospheric pressure and pneumothorax. Chest, 75, 650.

Scott, C.G., Berger, R. \& McKean, H.E. (1989) The role of of atmospheric pressure variation in the developement of spontaneous pneumothorax. Am. Rev. Respir. Dis., 139, 659-662.

Smit, H.J., Devillé, W.L., Schramel, F.M. \& Postmus, P.E. (1997) Spontaneous pneumothorax: predictable mini-epidemics? Lancet, 350, 1450.

Smit, H.J., Devillé, W.L., Schramel, F.M., Schreurs, J.M., Sutedja, T.G. \& Postmus, P.E. (1999) Atmospheric pressure changes and outdoor temperature changes in relation to spontaneous pneumothorax. Chest, 116, 676-681.

Suarez-Varel, M.M., Martinez-Selva, M.I., Llopis-Gonzalez, A., Martinez-Jimeno, J.L. \& Plaza-Valia, P. (2000) Spontaneous pneumothorax related with climatic characteristics in the Valencia area (Spain). Eur. J. Epidemiol, 16, 193-198. 